\title{
Transcriptomic Analysis of the Maize Mutant TC19 Identifies Genes Regulating the Development of Grain Size Through the IAA and BR Pathways
}

\section{Yanrong Zhang}

Qingdao Agricultural University

Fuchao Jiao

Qingdao Agricultural University

Jun Li

Qingdao Agricultural University

Yuhe Pei

Qingdao Agricultural University

Meiai Zhao

Qingdao Agricultural University

Xiyun Song

Qingdao Agricultural University

Xinmei Guo ( $\nabla$ xmguo2009@126.com )

Qingdao Agricultural University

\section{Research Article}

Keywords: maize, grain size, mutant, hormone, transcriptome

Posted Date: June 3rd, 2021

DOl: https://doi.org/10.21203/rs.3.rs-517785/v1

License: (9) (i) This work is licensed under a Creative Commons Attribution 4.0 International License.

Read Full License 


\section{Abstract}

Backgrounds: Grain size is a key factor in crop yield that gradually develops after pollination. However, few studies have reported gene expression patterns in maize grain development using mutants. To investigate the developmental mechanisms of grain size, we analyzed a large-grain mutant, named TC19, at the morphological and transcriptome level at five stages corresponding to days after pollination (DAP).

Results: After maturation, the grain length, width, and thickness in TC19 were greater than that in Chang $7-2$ (control) and increased by $3.57 \%, 8.80 \%$, and $3.88 \%$, respectively. Further analysis showed that grain width in TC19 was lower than in Chang 7-2 at 7, 14, and 21 DAP, but greater than that in Chang 7-2 at 28 and 35 DAP, indicating that 21 to 28 DAP was the critical stage for kernel width development. For all five stages, the concentrations of indole-3-acetic acid and brassinosteroids were significantly higher in TC19 than in Chang 7-2. Gibberellin was higher at 7, 14, and 21 DAP, and cytokinin was higher at 21 and 35 DAP, in TC19 than in Chang 7-2. Through transcriptome analysis at 14, 21, and 28 DAP, we identified 2987, 2647, and 3209 differentially expressed genes (DEGs) between TC19 and Chang 7-2. Gene Ontology analysis indicated that most of the grain size-related genes corresponded to three aspects, including cell components, molecular functions, and biological processes. The Kyoto Encyclopedia of Genes and Genomes pathway analysis showed that 77 DEGs were enriched in the plant hormone signal transduction pathway. We further analyzed several highly expressed candidate genes, including $A O 2$, $A R F 3$, and IAA15, which are involved in the synthesis of IAA; and DWF4 and XTH, which are involved in the synthesis of $B R$.

Conclusions: Our results elucidated the mechanisms of grain size development at the grain-filling stage and have potential application in maize breeding.

\section{Background}

Maize is an important human food, livestock feed, and bioenergy crop of great economic significance. Global maize production reached 1.1 billion tons in 2019 according to the Food and Agriculture Organization, providing a significant amount of food, feed, and bioenergy raw materials. The number of ears per unit area, number of grains per ear, and grain weight are the three main factors of maize yield [1]. Among these, grain weight is the primary factor affecting yield, because decreased grain weight cannot be compensated for by other yield factors. Grain size and filling degree are the main factors that affect grain weight, and thus grain size is an important trait affecting grain weight.

Maize seeds are composed of an embryo, endosperm, and seed coat. The maize embryo includes the germ, germ sheath, hypocotyl, radicle, sheath, and shield. The maize endosperm accounts for more than $80 \%$ of the volume and dry weight of the whole seed and is the most important component of maize seeds. The weight and quality of maize seeds are determined by the development, proliferation, and enrichment of endosperm cells. The maize endosperm is developed by the fusion of a male gamete with two polar nuclei. Maize endosperm development includes several stages: primary endosperm nuclear 
division, syncyte stage, syncyte cytochemistry, mitotic boom stage, nutrient storage stage, and dehydration and maturation stage [2].

Beneficial allelic variation provides the genetic basis for maize yield improvement [3]. The frequency of spontaneous mutations in plants is extremely low, with the mutation frequency of a single plant ranging from $1 / 10^{3}$ to $1 / 10^{4}$ and that of a single gene ranging from $1 / 10^{5}$ to $1 / 10^{6}$. Therefore, spontaneous mutations cannot ensure sufficient beneficial variations. By using physical and chemical mutagenesis technology, tens of thousands of mutant varieties of crops, vegetables, and flowers have been bred. Some genes associated with maize grain development have been identified using mutants, such as Opaque2 [4], defective kernel [5], shrunken 1 [6], and embryo defective [7].

Seed development is a complex process regulated by plant hormones [8]. At present, many plant hormone-related genes are known to play critical roles in grain development. Genes associated with cytokinin, brassinolide, auxin, abscisic acid, and gibberellin are crucial for grain size. OSKX2 (Gn1a) encodes a cytokinin oxidase, and the knocking down of OSKX2 increases the activity of the rice inflorescence meristem, regulating the number of grains per panicle [9]. Overexpression of $S G 1$ weakens BR signal transduction in the ear and significantly reduces grain length. At the same time, the internode of the mother plant becomes shorter [10]. Rice $D 11$ participates in encoding cytochrome $\mathrm{P} 450$, which is involved in the synthesis of brassinolide in rice. Dwarf 11 mutants are severely dwarfed with shorter grains [9].

RNA sequencing is an efficient transcriptomic technology for gene expression analysis [11]. Many genes have been identified as being involved in grain development using natural variations [12, 13]. Few studies, however, have reported gene expression patterns in maize grain development using mutants. TC19 is a large mutant that was selected from Chang 7 - 2 following Co60 gamma-ray radiation and that possesses significant differences in both grain size and weight. By using RNA sequencing, we analyzed the transcriptomic differences between TC19 and Chang 7-2 and identified potential genes related to grain size and grain weight.

\section{Methods}

\section{Plant growth and phenotyping}

The seeds of Chang 7 - 2 were obtained from the maize center of Qingdao Agricultural University. TC19 was originally generated after Co60-y radiation on Chang 7 - 2 background in the Song lab in Qingdao Agricultural University. The permission of seeds collection has been obtained. Chang 7- 2 and TC19 were sown in Sanya (SY, $\left.18^{\circ} 30^{\prime} \mathrm{N}, 108^{\circ} 47^{\prime} \mathrm{E}\right)$ in 2014 and 2015, and Jiaozhou (JZ, 36 $\left.04^{\prime} \mathrm{N}, 120^{\circ} 18^{\prime} \mathrm{E}\right)$ in 2015 and 2016. Single seeds were sown with a 3-meter row length, 0.6-meter row spacing, and 0.2-meter plant spacing, with 10 rows per material under normal field management practices. All plants were selfpollinated. Cobs were taken at 7 days, 14 days, 21 days, 28 days, and 35 days after pollination. Grains were isolated from the center of the cobs at the same growth stage. For each treatment, three cobs were 
selected, and when the maize was mature, they were single-ear harvested and dried naturally to a water content of about $13 \%$. Afterward, at least three ears were selected for measurement. Grains at the same growth stage and of the same shape were selected for measurement of kernel length, width, thickness, and 100-kernel weight. The data were analyzed using Excel 2016 and SPASS 2.0. We declare that all the collections of plant and seed specimens related to this study were performed in accordance with the relevant guidelines and regulations by Ministry of Agriculture (MOA) of the People's Republic of China.

\section{Determination of endogenous hormone content}

The maize inbred lines Chang $7-2$ and TC19 were sown in the Modern Agricultural Science and Technology Demonstration Park of Qingdao Agricultural University in 2016. After tasseling, they were all self-pollinated. Cobs were sampled at 7, 14, 21, 28, and 35 DAP. Grains were isolated from the center of the cobs at the same growth stage, and more than three cobs were sampled for each treatment.

Samples of $0.2 \mathrm{~g}-0.5 \mathrm{~g}$ were rinsed in ice-cold PBS $(0.05 \mathrm{~mol} / \mathrm{L}$ Tris-HCl, $\mathrm{pH}=7.4)$, wiped dry with filter paper, weighed accurately, and placed into a $5 \mathrm{~mL}$ homogenization tube. Four times the volume of homogenization medium was added to the homogenization tube at the ratio of weight $(\mathrm{mg})$ : volume $(\mathrm{mL})=1: 4$, and the tissue block was cut as soon as possible using small ophthalmic scissors in an ice water bath. A tissue masher was used for grinding the tissue at $10000-15000 \mathrm{r} / \mathrm{min}$. A small amount of tissue homogenate was taken for smearing (direct smear or staining), and the broken cells were observed under a microscope. The sample was then centrifuged at $4000 \mathrm{r} / \mathrm{min}$ for $10-15 \mathrm{~min}$, and the supernatant was used for determination.

Following dilution and addition of the standard product, we tested the blank wells and sample wells. Forty microliters of sample diluent was added to the test sample well on the enzyme-labeled plate, following which $10 \mu \mathrm{L}$ of the test sample was added. The plate was sealed with parafilm and incubated at $37^{\circ} \mathrm{C}$ for $30 \mathrm{~min}$. Twenty-fold concentrated washing liquid was diluted with distilled water 20 times and then set aside. The sealing plate membrane was carefully removed, the liquid was discarded, and the sample was spun dry. Each well was filled with the washing liquid and left to stand for $30 \mathrm{~s}$, following which it was discarded. This was repeated five times, following which the wells were patted dry. Then, 50 $\mu \mathrm{L}$ of enzyme-labeled reagent was added to each well, except for the blank wells. The plate was sealed with sealing film and incubated at $37^{\circ} \mathrm{C}$ for $30 \mathrm{~min}$. The sealing film was then carefully removed, the liquid was discarded, the wells were spun dry, and each well was filled with washing liquid and allowed to stand for $30 \mathrm{~s}$, following which the liquid was discarded. This was repeated five times, and the wells were patted dry. Fifty microliters of color developer A was first added to each well, followed by $50 \mu \mathrm{L}$ of color developer $B$, and then the plates were gently mixed, and the color was developed at $37^{\circ} \mathrm{C}$ for $15 \mathrm{~min}$ in the dark. Fifty microliters of stop solution was then added to each well to stop the reaction. The absorbance (OD value) of each well was measured at $450 \mathrm{~nm}$ wavelength.

Taking the concentration of the standard substance as the ordinate $(Y)$ and the OD value as the abscissa $(X)$, we calculated the polynomial quadratic regression equation of the standard curve. The OD value of the sample was substituted into the equation, and the sample concentration was calculated and 
multiplied by the dilution factor, which represents the actual concentration of the sample. In this experiment, the sample dilution factor was five times, and the quadratic regression equation of each hormone was as follows:

Gibberellin (GA3): $Y=0.4303+34.5196 X ;$

Auxin (IAA): $Y=-1.6192+32.3868 X$;

Cytokinin (CTK): $Y=1.1722+21.0967 X$;

Brassinolide (BR): $Y=6.8315+83.9345 X$.

\section{RNA isolation and sequencing}

At 14 DAP, 21 DAP, and 28 DAP, we sampled the grains from the center of the Chang $7-2$ and TC19 cobs for total RNA isolation. The grains were ground into powder in liquid nitrogen and placed in a $2 \mathrm{~mL}$ Eppendorf tube. We then added $1500 \mathrm{~mL}$ of the extraction reagent TRNzol-At, vortexed the mixture thoroughly, and then incubated the mixture at room temperature for $30 \mathrm{~min}$. The sample was then centrifuged at $12000 \mathrm{rpm}$ for $10 \mathrm{~min}$, following which the supernatant was transferred to a new RNasefree centrifuge tube. Three-hundred microliters of chloroform/isoamyl alcohol (24:1) was added, and the mixture was shaken at $15 \mathrm{rpm}$ for $20 \mathrm{~s}$ and incubated at room temperature for $15 \mathrm{~min}$. The sample was then centrifuged at $12000 \mathrm{rpm}$ at $4^{\circ} \mathrm{C}$ for $15 \mathrm{~min}$, and then $500 \mu \mathrm{L}$ of the supernatant was transferred to a new RNase-free centrifuge tube. An equal volume of isopropanol (pre-cooled at $-20^{\circ} \mathrm{C}$ ) was added to the tube, which was mixed well and then incubated at room temperature for $15 \mathrm{~min}$. Following centrifugation at $12000 \mathrm{rpm}$ for $10 \mathrm{~min}$ at $4^{\circ} \mathrm{C}$, the supernatant was discarded. One milliliter of $80 \%$ absolute ethanol $\left(-20^{\circ} \mathrm{C}\right.$ pre-cooled) was added to the centrifuge tube, which was shaken gently by hand to wash the precipitate and then centrifuged at $4^{\circ} \mathrm{C}$ and $12000 \mathrm{rpm}$ for $3 \mathrm{~min}$. Once the ethanol had completely evaporated, $40 \mu \mathrm{L}$ of RNase-free water was added and repeatedly mixed by pipetting to fully dissolve the RNA. Agarose gel electrophoresis was used to detect whether the total RNA was contaminated as well as the degree of RNA degradation. A Nanodrop was used to further detect the purity of the RNA (OD260/OD280), and a Qubit was used to accurately quantify the concentration of the RNA. An Agilent 2100 was used to accurately detect the integrity of the RNA.

\section{Construction of a cDNA library and computer sequencing}

After the RNA quality of the sample had been qualified, the mRNA was enriched with Oligo (dT) magnetic beads, and fragmentation buffer was added to the mRNA, six bases random hexamers, buffer, dNTPs, RNase $\mathrm{H}$, and DNA polymerase I were added to synthesize the second strand of CDNA, which was purified by a QiaQuick PCR kit and eluted with EB buffer. After end-repair, alkali addition Base A, and addition of sequencing adapters, the target size fragments were recovered by agarose gel electrophoresis, and PCR amplification was performed to complete the library preparation work. The constructed library was sequenced on an Illumina HiSeqTM 2500.

\section{Analysis of sequencing information}


We used base calling to convert the raw image data measured by the Illumina HiSeqTM 2500 into sequence data, namely raw reads. To ensure data quality and reduce data noise through data filtering, reads containing adapters and reads with a ratio of $\mathrm{N}$ greater than $10 \%$ and low-quality reads (the number of bases with a quality value of $Q \leq 20$ accounting for more than $50 \%$ of the entire read) were filtered to obtain high-quality clean reads for subsequent analysis. TopHat2 was used to align clean reads to the reference genome. The reference genome database used Ensembl Plants (http://plants.ensembl.org/Zea_mays/Info/Index). After the reads had been aligned to the genome, it was assembled using Cufflinks.

\section{Expression statistics}

We calculated the expression using the FPKM (Fragments Per Kilobase of transcript per Million mapped reads) method with the formula: $\mathrm{FPKM}=106 \mathrm{C} /(\mathrm{NL} / 103)$, where $\mathrm{FPKM}(\mathrm{A})$ is the expression level of gene $A ; C$ is the number of sequenced fragments compared to gene $A ; N$ is the total number of sequenced fragments compared to the reference gene; and $L$ is the number of bases of gene $A$.

\section{Repeatability test}

Biological replication is necessary for any biological experiment, and high-throughput sequencing technology is no exception. Biological repetition has two main purposes: one is to prove that the involved biological experiment operations can be repeated with little variation, and the other is needed for subsequent differential gene analysis. The correlation of gene expression levels between samples is an important indicator for testing the reliability of experiments and the rationality of sample selection. The closer the correlation coefficient is to 1 , the higher the similarity of the expression patterns between samples.

\section{Difference analysis}

We calculated the expression difference of a gene in different samples according to the FPKM value of the gene. The criteria for DEGs was a log2foldchange $\geq 1$ (the multiple of difference is greater than or equal to 2) and a $P$-value of $\leq 0.05$. Significant differences between paired samples or between groups of RNA-seq data were processed using edge $\mathrm{R}$. For detailed instructions on edge $\mathrm{R}$, please refer to: http://www.bioconductor.org/packages/release/bioc/html/edgeR.html. The expression patterns of genes in different samples were analyzed, and the DEGs and co-expressed differential genes between the samples were screened, and on this basis, further genes related to the target traits were identified.

GO function analysis

We mapped the DEGs to each term in the GO database (http://www.geneontology.org/) and calculated the number of genes in each term to obtain a list of genes with GO functions and gene item statistics. We 
then applied a hypergeometric test to determine the GO entries that were significantly enriched in the DEGs compared with the whole genomic background.

Kyoto Encyclopedia of Genes and Genomes enrichment analysis

In organisms, different genes coordinate with each other to perform their biological functions. Pathwaybased analysis helps us to further understand the biological functions of genes. Kyoto Encyclopedia of Genes and Genomes (KEGG) is the main public database on pathways [90]. Pathway-significant enrichment analysis uses KEGG pathways as the unit and applies hypergeometric tests to determine pathways that are significantly enriched in DEGs compared with the entire genome background. Pathwaysignificant enrichment can determine the most important biochemical metabolic pathways and signal transduction pathways associated with DEGs.

\section{Results}

\section{Grain size and grain weight}

To elucidate the consequence of mutations on grain size development, we performed morphological analysis using TC19 and Chang 7 - 2 in two locations for two years. We found that the length, width, thickness, and 100-kernel weight of the mature seeds of TC19 were significantly greater than in Chang 7

- 2. Grain length in TC19 increased by $3.57 \%$, grain width increased by $8.8 \%$, and grain thickness increased by $3.88 \%$ compared with Chang $7-2$. The grain volume and 100-grain weight of TC19 increased by $18.75 \%$ and $16.94 \%$, respectively (Fig. 1). However, ear length and grain weight in TC19 were significantly lower than in Chang $7-2$ (Table 1). These results indicated that ear weight may decrease when kernel size is increased. 
Table 1

Statistics data for grain and ear in chang7-2 and TC19

\begin{tabular}{|lll|}
\hline traits & chang7-2 & TC19 \\
\hline Grian length $(\mathrm{mm})$ & $9.23 \pm 0.59$ & $9.56 \pm 0.74^{\star *}$ \\
\hline Grain width $(\mathrm{mm})$ & $7.5 \pm 0.53$ & $8.16 \pm 0.81^{\star \star}$ \\
\hline Grain thickness $(\mathrm{mm})$ & $4.64 \pm 0.61$ & $4.82 \pm 0.64^{\star \star}$ \\
\hline Grain length/width & $1.23 \pm 0.12$ & $1.17 \pm 0.11^{\star}$ \\
\hline Grain volume $\left(\mathrm{cm}^{3}\right)$ & $0.32 \pm 0.002$ & $0.38 \pm 0.0032^{\star}$ \\
\hline 100 kernel weight $(\mathrm{g})$ & $21.45 \pm 0.72$ & $25.08 \pm 0.55^{\star \star}$ \\
\hline Kernel row number & $14 \pm 0.5$ & $16 \pm 0.8$ \\
\hline Ear length $(\mathrm{cm})$ & $12.69 \pm 1.07^{\star \star}$ & $7.84 \pm 1.16$ \\
\hline Ear width $(\mathrm{cm})$ & $4.06 \pm 0.09$ & $4.53 \pm 0.05^{\star *}$ \\
\hline Ear weight $(\mathrm{g})$ & $93.94 \pm 4.85^{\star \star}$ & $70.76 \pm 3.38$ \\
\hline
\end{tabular}

Environmental factors have a great influence on plant growth and development. In this study, the grain length, grain width, grain thickness, and 100-kernel weight of Chang $7-2$ and TC19 were influenced significantly by the environment. However, the grain length, grain width, grain thickness, and 100-kernel weight of TC19 were significantly greater than those of Chang $7-2$ in every environment (Fig. 1), indicating that grain size is mainly controlled by genetic factors.

Grain width changed most obviously between the mature seeds of TC19 and Chang $7-2$. To determine the stage at which this difference occurred, we measured the grain width from 7 to 35 days after pollination (DAP) every $7 \mathrm{~d}$. Before $21 \mathrm{DAP}$, the grain width of TC19 was significantly smaller than that of Chang $7-2$. However, after 28 DAP, the grain width of TC19 was significantly larger than that of Chang 7 - 2. The grain width of TC19 increased rapidly from 14 to 28 DAP, which ultimately contributed to the difference between TC19 and Chang 7 - 2 (Fig. 2).

\section{Endogenous hormones}

Plant endogenous hormones, such as indole-3-acetic acid (IAA), brassinosteroids (BR), gibberellins (GAs), and cytokinin (CTK), play important roles in the regulation of seed size. To elucidate the effects of hormones between TC19 and Chang $7-2$, we detected the concentrations of IAA, BR, GA3, and CTK at five stages after pollination (Table S1). The concentration of IAA in TC19 peaked at 21 DAP, following which it decreased slightly at 28 and 35 DAP. The concentration of IAA in Chang $7-2$ also peaked at 21 DAP, reaching its lowest level at 28 DAP and then increasing slightly at 35 DAP (Fig. 3A). The concentration pattern of BR was similar to that of IAA (Fig. 3D). The concentrations of IAA and BR in TC9 were higher than those in Chang $7-2$ at all five stages (Fig. 3A and 3D). The concentration of GA3 decreased in TC19 but increased in Chang $7-2$ from 7 to 35 DAP. The concentration of GA3 in TC19 was 
significantly higher than that in Chang $7-2$ from 7 to 21 DAP and was no longer significant after 28 DAP (Fig. 3B). The concentration of CTK in TC19 was higher than that in Chang 7-2 at 21 DAP, but it was not significantly different at 7, 14, 28, and 35 DAP (Fig. 3C).

\section{Sequencing data quality assessment}

First, all of the parameters for RNA quality met the library construction standards. After Illumina sequencing, the Q30 base quality value of all the samples was higher than $90 \%$, and the GC content was around $55 \%$ (Table S2). After alignment, more than $70 \%$ of the reads in each sample were aligned to the reference B73 genome sequence. The correlation coefficient between three replicates of the samples exceeded 0.97 (Table 2). These results confirmed that the data were reliable.

\section{Differential gene statistics}

We screened differentially expressed genes (DEGs) between Chang $7-2$ and TC19 at different stages after pollination. There were 2987, 2647, and 3209 DEGs at 14, 21, and 28 DAP, respectively. Compared with Chang 7-2,1201 genes increased and 1786 genes decreased in TC19 at 14 DAP. A total of 1647 genes increased and 1000 genes decreased in TC19 at 21 DAP, and 1995 genes increased and 1214 genes decreased in TC19 at 28 DAP (Fig. 8A). 
Table 2

Reads of samples

\begin{tabular}{|c|c|c|c|c|c|}
\hline Sample & $\begin{array}{l}\text { Total } \\
\text { Reads }\end{array}$ & $\begin{array}{l}\text { Unmapped } \\
\text { Reads }\end{array}$ & $\begin{array}{l}\text { Unique Mapped } \\
\text { Reads }\end{array}$ & $\begin{array}{l}\text { Multiple Mapped } \\
\text { reads }\end{array}$ & $\begin{array}{l}\text { Mapping } \\
\text { Ratio }\end{array}$ \\
\hline CK-1-1 & 29162394 & $27.44 \%$ & $71.10 \%$ & $1.46 \%$ & $72.56 \%$ \\
\hline CK-1-2 & 30751438 & $27.47 \%$ & $71.11 \%$ & $1.42 \%$ & $72.53 \%$ \\
\hline CK-1-3 & 31965840 & $27.63 \%$ & $70.84 \%$ & $1.53 \%$ & $72.37 \%$ \\
\hline CK-2-1 & 31285730 & $27.34 \%$ & $71.24 \%$ & $1.42 \%$ & $72.66 \%$ \\
\hline CK-2-2 & 20401138 & $27.23 \%$ & $71.32 \%$ & $1.45 \%$ & $72.77 \%$ \\
\hline CK-2-3 & 27313866 & $26.87 \%$ & $71.66 \%$ & $1.47 \%$ & $73.13 \%$ \\
\hline CK-3-1 & 33449740 & $28.13 \%$ & $70.51 \%$ & $1.36 \%$ & $71.87 \%$ \\
\hline CK-3-2 & 30453088 & $28.65 \%$ & $70.14 \%$ & $1.21 \%$ & $71.35 \%$ \\
\hline CK-3-3 & 25112472 & $28.19 \%$ & $70.51 \%$ & $1.30 \%$ & $71.81 \%$ \\
\hline $\begin{array}{l}\text { TC19- } \\
1-1\end{array}$ & 29571446 & $25.57 \%$ & $73.22 \%$ & $1.21 \%$ & $74.43 \%$ \\
\hline $\begin{array}{l}\text { TC19- } \\
1-2\end{array}$ & 27076892 & $24.64 \%$ & $74.14 \%$ & $1.21 \%$ & $75.36 \%$ \\
\hline $\begin{array}{l}\text { TC19- } \\
1-3\end{array}$ & 31937200 & $25.21 \%$ & $73.64 \%$ & $1.15 \%$ & $74.79 \%$ \\
\hline $\begin{array}{l}\text { TC19- } \\
2-1\end{array}$ & 35862450 & $26.90 \%$ & $71.87 \%$ & $1.23 \%$ & $73.10 \%$ \\
\hline $\begin{array}{l}\text { TC19- } \\
2-2\end{array}$ & 31611148 & $27.07 \%$ & $71.78 \%$ & $1.16 \%$ & $72.93 \%$ \\
\hline $\begin{array}{l}\text { TC19- } \\
2-3\end{array}$ & 28865264 & $26.73 \%$ & $72.12 \%$ & $1.16 \%$ & $73.27 \%$ \\
\hline $\begin{array}{l}\text { TC19- } \\
3-1\end{array}$ & 33666020 & $27.68 \%$ & $71.20 \%$ & $1.12 \%$ & $72.32 \%$ \\
\hline $\begin{array}{l}\text { TC19- } \\
3-2\end{array}$ & 34859588 & $28.51 \%$ & $70.41 \%$ & $1.08 \%$ & $71.49 \%$ \\
\hline $\begin{array}{l}\text { TC19- } \\
3-3\end{array}$ & 34154116 & $28.30 \%$ & $70.63 \%$ & $1.08 \%$ & $71.70 \%$ \\
\hline
\end{tabular}

\section{Gene Ontology function analysis}

Through Gene Ontology (GO) analysis, the DEGs between Chang 7 - 2 and TC19 were distributed mainly in three categories, including cell components, molecular functions, and biological processes (Fig. 4A). At 21 DAP, the DEGs were enriched in the cellular components GO: 0000785 (chromatin) and G0: 0005576, 
GO: 0044427, GO: 0071944, GO: 0005694 (chromatin, chromosomes, extracellular regions). In terms of molecular functions, the DEGs were mainly enriched in GO: 0016627 and GO: 0469983 (oxidoreductase activity and protein dimerization) (Fig. 4B).

At 28 DAP, the DEGs were enriched in cellular components (G0:0005576), and the genes enriched in terms of molecular function included G0:0016491 (oxidoreductase activity), G0:0046906 (tetrapyrrole combination), GO: 0003824 (catalytic activity), GO: 0061134 (peptidase regulation activity), GO: 0061135 (endopeptidase regulation activity), GO: 0016798 (hydrolase activity), GO: 0004866 (peptidase inhibitor activity), GO: 0004857 (enzyme inhibitor activity), and GO: 0003700 (activation of transcription factors). Genes in biological processes were enriched in GO: 0010466, GO: 0052547, G0: 0054861, G0: 0030162, GO: 0051346, GO: 0043086, GO: 0016052, GO: 0032269, GO: 0051248, GO: 0044092, and GO: 0000272; these $\mathrm{GO}$ terms mainly included the decomposition of starch or polysaccharides and some regulation (Fig. 4C).

\section{Kyoto Encyclopedia of Genes and Genomes enrichment analysis}

To determine the biochemical metabolic pathways and signal transduction pathways associated with the DEGs, we performed pathway-significant enrichment analysis. The results showed that the DEGs at 14 DAP were enriched mainly in the phenylpropane biosynthesis pathway, plant hormone signal transduction, phenylalanine metabolism, and starch sucrose metabolism pathway (Fig. 5). The DEGs at 21 DAP were enriched mainly in endoplasmic reticulum protein processing, plant hormone signal transduction, phenylpropanoid biosynthesis, and a-linolenic acid metabolic pathways (Fig. 6). The DEGs at 28 DAP were enriched mainly in phenylpropanoid biosynthesis, plant hormone signal transduction, brassinosteroid synthesis, and a-linolenic acid metabolism (Fig. 7). Above all, the DEGs in the hormone signal transduction pathway were significantly enriched. This indicated that the signal transduction pathway may play an important role in seed development.

\section{Genes enriched in hormone signal transduction}

We found a total of 77 DEGs related to the hormone signal transduction pathway (Fig. 8B). Among them, 27 genes were involved in the IAA signal transduction pathway; five genes were involved in the BR signal transduction pathway; seven genes were involved in the CTK signal transduction pathway; two genes were involved in the GA signal transduction pathway; six genes were involved the abscisic acid (ABA) signal transduction pathway; nine genes were involved in the ethylene (ET) signal transduction pathway; 11 genes were involved in the jasmonic acid (JA) signal transduction pathway; and 10 genes were involved in the SA signal transduction pathway.

\section{Genes related to IAA synthesis and signal transduction}


We detected 27 DEGs involved in the IAA signal transduction pathway. Cluster analysis confirmed that these genes exhibited different expression patterns between Chang $7-2$ and TC19 (Fig. 8C). We further detected two genes, namely Zm00001d012731 (ARF3) and Zm00001d039624 (IAA15), with high expression levels. At all three stages, the expression level of ARF3 in TC19 was higher than that in Chang 7 - 2. From 14 to 28 DAP, the expression of ARF3 was significantly increased in TC19, whereas it increased only slightly in Chang 7-2 (Fig. 9A). In all three periods, the expression of IAA15 in Chang 7-2 was higher than that of TC19, and there was no change between the three periods. Zm00001d034388 (AO2) in TC19 was higher than that of Chang 7- 2 in all three periods (Fig. 9B). The expression of AO2 increased in the three periods, increasing rapidly in TC19 but slowly in Chang 7 - 2 (Fig. 9C).

\section{Genes related to brassinolide biosynthesis and signal transduction}

Endogenous hormone analysis showed that the BR concentrations of Chang $7-2$ and TC19 differed significantly at all five stages of grain development. Analysis of the BR biosynthesis pathway indicated that ZM00001d003349 (DWF4) and Zm00001d014617 (XTH) were more highly expressed in TC19 than in Chang 7-2 (Fig. 9D and 9E).

\section{Discussion}

In this study, we used the TC19 maize mutant, which had been screened after Co60-y-ray irradiation and had been self-pollinated for multiple generations on the background of B73. Compared with Chang $7-2$, the grain length, grain width, grain thickness, and 100-kernel weight of TC19 were significantly increased, whereas the ear length and grain weight were reduced. Kernel number per grain, 100-kernel weight, and ear number are components of maize yield. The phenomenon of improved grain weight with reduced yield has been observed previously [14]. Some quantitative trait loci (QTLs) have been found to affect the balance between the kernel and ear $[15,16]$. Thus, the relationship between kernel, ear per plant, and field conditions should be carefully considered in plant breeding.

The grain type and grain weight of maize seeds not only are controlled by genetic factors, but also are affected by environmental factors, such as temperature, moisture, disease, and insect pests. To explore the difference between the grains of Chang $7-2$ and TC19, we analyzed the grain length, grain width, grain thickness, and 100-kernel weight under multiple different environmental conditions. The results showed that environment had a great effect on the grain size of maize. However, the grain size and weight of TC19 under each environmental condition were always higher than in Chang $7-2$, indicating that grain development mainly is genetically controlled. This is consistent with previous studies [17].

In this study, grain width was the main contributor to the difference in grain size between Chang $7-2$ and TC19. The grain width increased fastest in TC19 at 14-28 DAP, at which stage it exceeded Chang $7-2$, indicating that 14-28 DAP is an important period for grain enlargement. This stage is the grain-filling stage [18], and thus the grain-filling stage is critical for grain width. 
Plant hormones are one of the most important factors affecting the growth and development of seeds [19]. Many genes related to plant hormones have been confirmed to play key roles in regulating plant kernel development [20]. In recent years, it has been discovered that cytokinin and brassinolide play a vital role in regulating seed size, and in addition, auxin, $A B A$, and gibberellin have regulatory effects on seed development to a certain extent [21]. In this study, we detected changes in IAA, GA3, CTK, and BR during grain development in Chang $7-2$ and TC19. In all periods, the concentrations of IAA and BR in TC19 were significantly higher than in Chang $7-2$. The content of GA3 was significantly higher in TC19 than in Chang 7 - 2 in the first three stages, but not significantly so in the latter two periods. The content of CTK differed significantly only at 21 DAP, being significantly higher in TC19 than in Chang 7 - 2, and the difference between the two maize varieties was not significant at the other periods. We propose that auxin and brassinolide may have contributed significantly to the increased size of the TC19 grains.

Through transcriptomics analysis of the grains of Chang $7-2$ and TC19 at 14 days, 21 days, and 28 days after pollination, we found that the signal transduction pathway of plant hormones may have a notable influence on the grain size. Further screening of the DEGs revealed that five genes exhibited high expression levels, including ARF3, IAA15, AO2, DWF4, and XTH. Among them, ARF3, IAA15, and AO2 are related to the IAA biosynthesis or signal transduction pathway, and DWF4 and $X T H$ are related to the BR biosynthesis or signal transduction pathway [22]. IAA15 is a member of the AUX/IAA gene family [23], and $A R F 3$ is a member of the ARF family [24]. The Aux/IAA protein, as a type of transcription inhibitor, has been proven to play an extremely important role in the auxin signal transduction pathway. The auxin response requires the degradation of the Aux/IAA inhibitor, which causes the release of the ARF transcription factor that interacts with it and regulates the target gene. In this experiment, IAA15 was highly expressed in Chang $7-2$, whereas $A R F 3$ was lowly expressed in Chang $7-2$, indicating that IAA signal transduction in Chang 7 - 2 was inhibited compared with TC19. AO2 encodes 3-indole acetaldehyde oxidase, which is a key enzyme in the indolepyruvate pathway; the latter being the main pathway through which higher plants synthesize IAA. In this study, the expression of the AO2 gene in TC19 was higher than in Chang $7-2$ in the three periods, indicating that TC19 grains have higher levels of IAA, which was consistent with the endogenous hormone measurement results. DWF4 encodes sterol C-22a hydroxylase. Sterol C-22a hydroxylase acts as the rate-limiting link in the process of BR biosynthesis. A high expression of DWF4 increases the BR content in grains. In this study, the expression level of DWF4 in TC19 was always higher than that in Chang $7-2$, which was consistent with the higher BR content in TC19. XTHencodes xyloglucan endotransglycosidase/hydrolase, which is a cell wall relaxase and a key enzyme in plant cell wall remodeling. Studies have shown that $X T H s$ play roles in cell volume growth, and their expression is induced by brassinolide. The higher expression level of $X T H$ is consistent with the higher BR content in TC19. DWF4 may have an indirect regulatory effect on the $X T H$ gene-an aspect that could be further studied in the future (Fig. 10).

In addition to the genes related to auxin and brassinolide, some genes related to other hormones were differentially expressed between the two maize lines, but these genes may be involved in the process of grain development because of their low expression levels. The molecular regulatory mechanism of plant seed size is complex, and many genes and metabolites are involved in this process. The regulation of 
seed size by hormones forms only a part of this process. To study the regulatory mechanisms of seed size, the relationships and interactions between different substances or different genes need to be explored.

\section{Conclusion}

After maturation, the grain length, width, and thickness in TC19 were greater than that in Chang $7-2$ (control). Grain width in TC19 was greater than that in Chang 7-2 at 28 days after pollination. The concentrations of indole-3-acetic acid, brassinosteroids, gibberellin and cytokinin were significantly higher in TC19 than in Chang 7 - 2. There were 2987, 2647, and 3209 differentially expressed genes (DEGs) identified between TC19 and Chang $7-2$ at 14, 21, and 28 DAP, respectively. GO and KEGG analysis showed that 77 DEGs were enriched in the plant hormone signal transduction pathway, such as $A O 2$, ARF3, IAA15, DWF4 and XTH.

\section{Abbreviations}

DAP: days after pollination; IAA: indole-3-acetic acid; BR: brassinosteroids; Gas: gibberellins; CTK: cytokinin; DEGs: differentially expressed genes; ARF3: AUXIN RESPONSE FACTOR 3; IAA15 - Auxinresponsive protein IAA15; AO2: Aldehyde oxidase 2; DWF4: dwarf4; XTH: xyloglucan endotransglycosidase/hydrolase

\section{Declarations}

\section{Ethics approval and consent to participate}

Not Applicable.

\section{Consent for publication}

Not applicable.

\section{Availability of data and materials}

The raw sequence data are available in the NCBI Sequence Read Archive (SRA) repository. The accession number is PRJNA724904, the website link is https://dataview.ncbi.nlm.nih.gov/object/PRJNA724904. All data supporting the conclusions of this article are included in the article and its additional files.

\section{Competing interests}

The authors declare that they have no conflicts of interest.

\section{Funding}


This work was supported by the National Key Research and Development Program of China (2016YFD0102104), the National Natural Science Foundation of China (Grant No. 31201218), and Taishan Scholars Project (NO. tsqn201909134).

\section{Authors' contributions}

$X G$ and $X S$ designed the study. YZ performed the experiments. $Y Z$ and $F J$ analyzed the data and wrote the manuscript. All authors read and approved the final manuscript.

\section{Acknowledgments}

We thank all the colleagues in our laboratory, and thank LetPub (www.letpub.com) for its linguistic assistance during the preparation of this manuscript.

\section{Author information}

Yanrong Zhang and Fuchao Jiao contributed equally to this work.

\section{Affiliations}

College of Agronomy, Qingdao Agricultural University, Qingdao 266109, China

Yanrong Zhang, Fuchao Jiao, Jun Li, Yuhe Pei, Xiyun Song, Xinmei Guo

College of Life Science, Qingdao Agricultural University, Qingdao 266109, China

Meiai Zhao

Key Laboratory of Major Crop Germplasm Innovation and Application in Qingdao, Qingdao 266109, China Yanrong Zhang, Fuchao Jiao, Jun Li, Yuhe Pei, Meiai Zhao, Xiyun Song, Xinmei Guo

\section{References}

1. Lu X, Zhou Z, Yuan Z, Zhang C, Hao Z, Wang Z, Li M, Zhang D, Yong H, Han J, Li X, Weng J. Genetic Dissection of the General Combining Ability of Yield-Related Traits in Maize. Front Plant Sci. 2020 Jul 22;11:788. doi: 10.3389/fpls.2020.00788. PMID: 32793248; PMCID: PMC7387702.

2. Olsen OA. The Modular Control of Cereal Endosperm Development. Trends Plant Sci. 2020 Mar;25(3):279-290. doi: 10.1016/j.tplants.2019.12.003. Epub 2020 Jan 16. PMID: 31956036.

3. Liang Y, Liu HJ, Yan J, Tian F. Natural Variation in Crops: Realized Understanding, Continuing Promise. Annu Rev Plant Biol. 2021 Jan 22. doi: 10.1146/annurev-arplant-080720-090632. Epub ahead of print. PMID: 33481630.

4. MERTZ ET, BATES LS, NELSON OE. MUTANT GENE THAT CHANGES PROTEIN COMPOSITION AND INCREASES LYSINE CONTENT OF MAIZE ENDOSPERM. Science. 1964 Jul 17;145(3629):279 - 80. 
doi: 10.1126/science.145.3629.279. PMID: 14171571.

5. Neuffer MG, Sheridan WF. Defective kernel mutants of maize. I. Genetic and lethality studies. Genetics. 1980 Aug;95(4):929-44. PMID: 17249053; PMCID: PMC1214277.

6. Cobb BG, Hannah LC. Development of wild type, shrunken-1 and shrunken-2 maize kernels grown in vitro. Theor Appl Genet. 1983 Apr;65(1):47-51. doi: 10.1007/BF00276261. PMID: 24263200.

7. Li C, Shen Y, Meeley R, McCarty DR, Tan BC. Embryo defective 14 encodes a plastid-targeted cGTPase essential for embryogenesis in maize. Plant J. 2015 Nov;84(4):785 - 99. doi: 10.1111/tpj.13045. PMID: 26771182.

8. Figueiredo DD, Köhler C. Auxin: a molecular trigger of seed development. Genes Dev. 2018 Apr 1;32(7-8):479-490. doi: 10.1101/gad.312546.118. PMID: 29692356; PMCID: PMC5959232.

9. Tanabe S, Ashikari M, Fujioka S, Takatsuto S, Yoshida S, Yano M, Yoshimura A, Kitano H, Matsuoka M, Fujisawa Y, Kato H, Iwasaki Y. A novel cytochrome P450 is implicated in brassinosteroid biosynthesis via the characterization of a rice dwarf mutant, dwarf11, with reduced seed length. Plant Cell. 2005 Mar;17(3):776-90. doi: 10.1105/tpc.104.024950. Epub 2005 Feb 10. PMID: 15705958; PMCID: PMC1069698.

10. Nakagawa H, Tanaka A, Tanabata T, Ohtake M, Fujioka S, Nakamura H, Ichikawa H, Mori M. Short grain1 decreases organ elongation and brassinosteroid response in rice. Plant Physiol. 2012 Mar;158(3):1208-19. doi: 10.1104/pp.111.187567. Epub 2011 Dec 30. PMID: 22209874; PMCID: PMC3291246.

11. Owens NDL, De Domenico E, Gilchrist MJ. An RNA-Seq Protocol for Differential Expression Analysis. Cold Spring Harb Protoc. 2019 Jun 3;2019(6). doi: 10.1101/pdb.prot098368. PMID: 30952685.

12. Doll NM, Just J, Brunaud V, Caïus J, Grimault A, Depège-Fargeix N, Esteban $E$, Pasha $A$, Provart $N J$, Ingram GC, Rogowsky PM, Widiez T. Transcriptomics at Maize Embryo/Endosperm Interfaces Identifies a Transcriptionally Distinct Endosperm Subdomain Adjacent to the Embryo Scutellum. Plant Cell. 2020 Apr;32(4):833-852. doi: 10.1105/tpc.19.00756. Epub 2020 Feb 21. PMID: 32086366; PMCID: PMC7145466.

13. Du X, Fang T, Liu Y, Huang L, Zang M, Wang G, Liu Y, Fu J. Transcriptome Profiling Predicts New Genes to Promote Maize Callus Formation and Transformation. Front Plant Sci. 2019 Dec 20;10:1633. doi: 10.3389/fpls.2019.01633. PMID: 31921272; PMCID: PMC6934073.

14. Zhai H, Feng Z, Du X, Song Y, Liu X, Qi Z, Song L, Li J, Li L, Peng H, Hu Z, Yao Y, Xin M, Xiao S, Sun Q, $\mathrm{Ni}$ Z. A novel allele of TaGW2-A1 is located in a finely mapped QTL that increases grain weight but decreases grain number in wheat (Triticum aestivum L.). Theor Appl Genet. 2018 Mar;131(3):539553. doi: 10.1007/s00122-017-3017-y. Epub 2017 Nov 17. PMID: 29150697; PMCID: PMC5814529.

15. Huo D, Ning Q, Shen X, Liu L, Zhang Z. QTL Mapping of Kernel Number-Related Traits and Validation of One Major QTL for Ear Length in Maize. PLoS One. 2016 May 13;11(5):e0155506. doi: 10.1371/journal.pone.0155506. PMID: 27176215; PMCID: PMC4866764.

16. Wang J, Lin Z, Zhang X, Liu H, Zhou L, Zhong S, Li Y, Zhu C, Lin Z. krn1, a major quantitative trait locus for kernel row number in maize. New Phytol. 2019 Aug;223(3):1634-1646. doi: 
10.1111/nph.15890. Epub 2019 Jun 6. PMID: 31059135.

17. Martinez-Feria RA, Licht MA, Ordóñez RA, Hatfield JL, Coulter JA, Archontoulis SV. Evaluating maize and soybean grain dry-down in the field with predictive algorithms and genotype-by-environment analysis. Sci Rep. 2019 May 9;9(1):7167. doi: 10.1038/s41598-019-43653-1. PMID: 31073235; PMCID: PMC6509253.

18. Chen J, Zeng B, Zhang M, Xie S, Wang G, Hauck A, Lai J. Dynamic transcriptome landscape of maize embryo and endosperm development. Plant Physiol. 2014 Sep;166(1):252-64. doi: 10.1104/pp.114.240689. Epub 2014 Jul 18. PMID: 25037214 ; PMCID: PMC4149711.

19. Penfield S. Seed dormancy and germination. Curr Biol. 2017 Sep 11;27(17):R874-R878. doi: 10.1016/j.cub.2017.05.050. PMID: 28898656.

20. Ge F, Luo X, Huang X, Zhang Y, He X, Liu M, Lin H, Peng H, Li L, Zhang Z, Pan G, Shen Y. Genome-wide analysis of transcription factors involved in maize embryonic callus formation. Physiol Plant. 2016 Dec;158(4):452-462. doi: 10.1111/ppl.12470. Epub 2016 Jun 9. PMID: 27194582.

21. Chen L, Li YX, Li C, Shi Y, Song Y, Zhang D, Wang H, Li Y, Wang T. The retromer protein ZmVPS29 regulates maize kernel morphology likely through an auxin-dependent process(es). Plant Biotechnol J. 2020 Apr;18(4):1004-1014. doi: 10.1111/pbi.13267. Epub 2019 Oct 17. PMID: 31553822; PMCID: PMC7061865.

22. Shahnejat-Bushehri S, Tarkowska D, Sakuraba Y, Balazadeh S. Arabidopsis NAC transcription factor JUB1 regulates GA/BR metabolism and signalling. Nat Plants. 2016 Feb 29;2:16013. doi: 10.1038/nplants.2016.13. PMID: 27249348.

23. Kim SH, Bahk S, An J, Hussain S, Nguyen NT, Do HL, Kim JY, Hong JC, Chung WS. A Gain-of-Function Mutant of IAA15 Inhibits Lateral Root Development by Transcriptional Repression of LBD Genes in Arabidopsis. Front Plant Sci. 2020 Aug 12;11:1239. doi: 10.3389/fpls.2020.01239. PMID: 32903377; PMCID: PMC7434933.

24. Vial-Pradel S, Keta S, Nomoto M, Luo L, Takahashi H, Suzuki M, Yokoyama Y, Sasabe M, Kojima S, Tada Y, Machida Y, Machida C. Arabidopsis Zinc-Finger-Like Protein ASYMMETRIC LEAVES2 (AS2) and Two Nucleolar Proteins Maintain Gene Body DNA Methylation in the Leaf Polarity Gene ETTIN (ARF3). Plant Cell Physiol. 2018 Jul 1;59(7):1385-1397. doi: 10.1093/pcp/pcy031. PMID: 29415182.

\section{Figures}




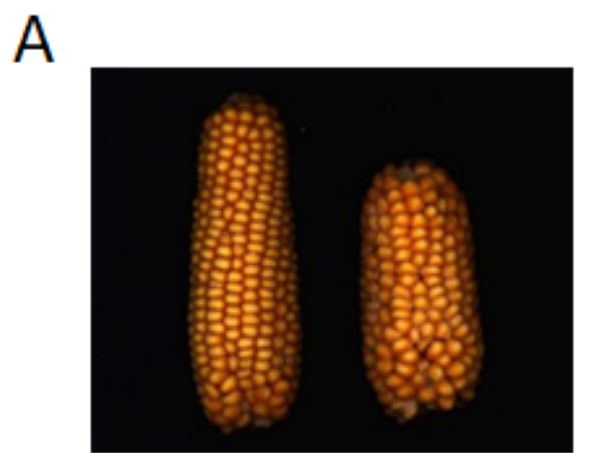

Chang7-2 TC19

C

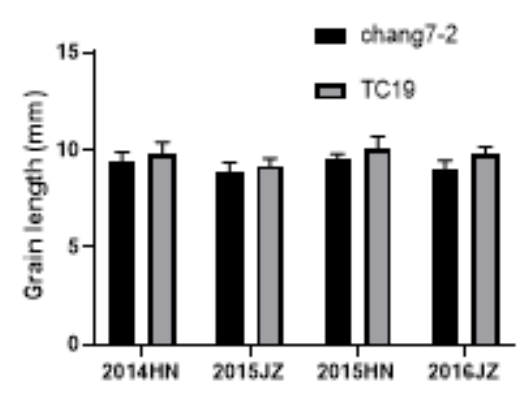

E

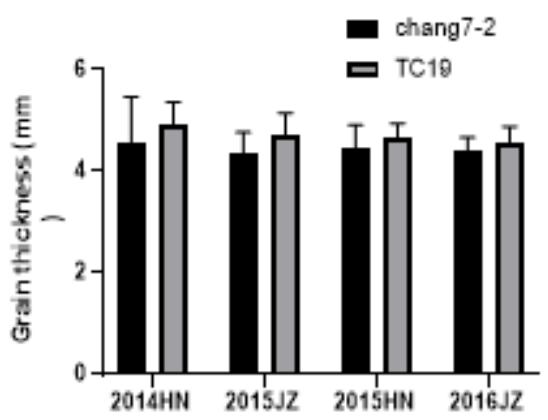

B

Chargy $7-2$

TC19

Chang7-2

TC19
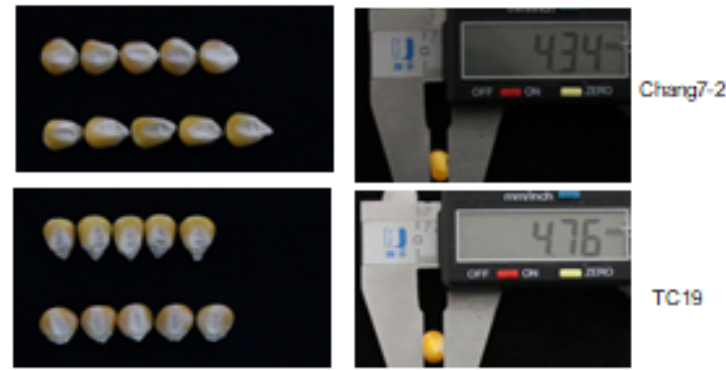

D

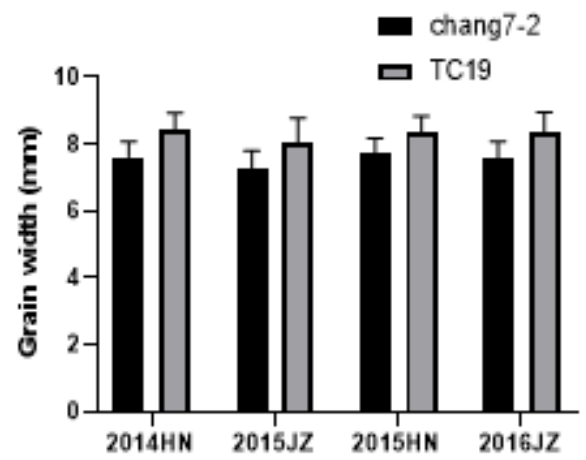

$\mathrm{F}$

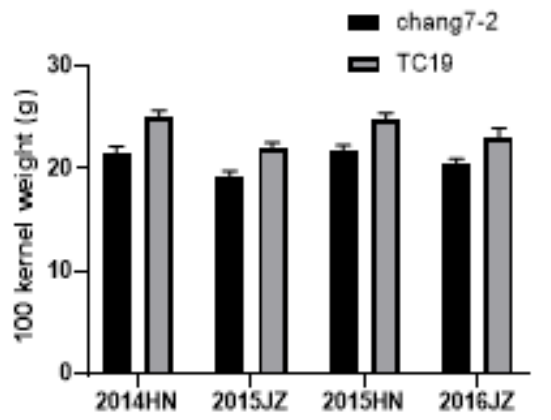

Figure 1

The differences in grain size between Chang 7-2 and TC19. A and B, photographs of Chang 7-2 and TC19. C-F, statistics for grain size between Chang 7-2 and TC19. 
A

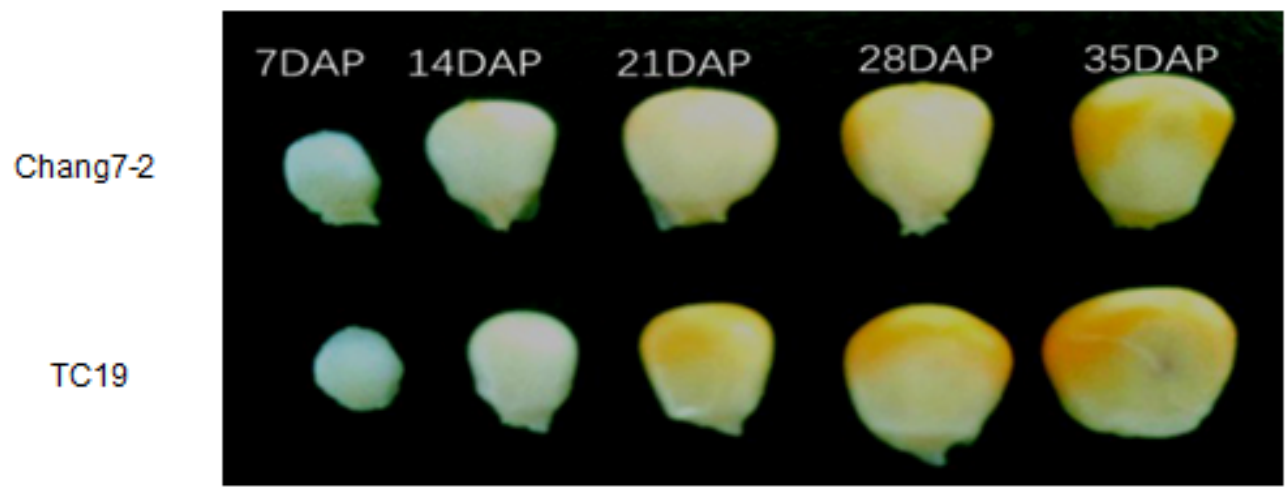

B

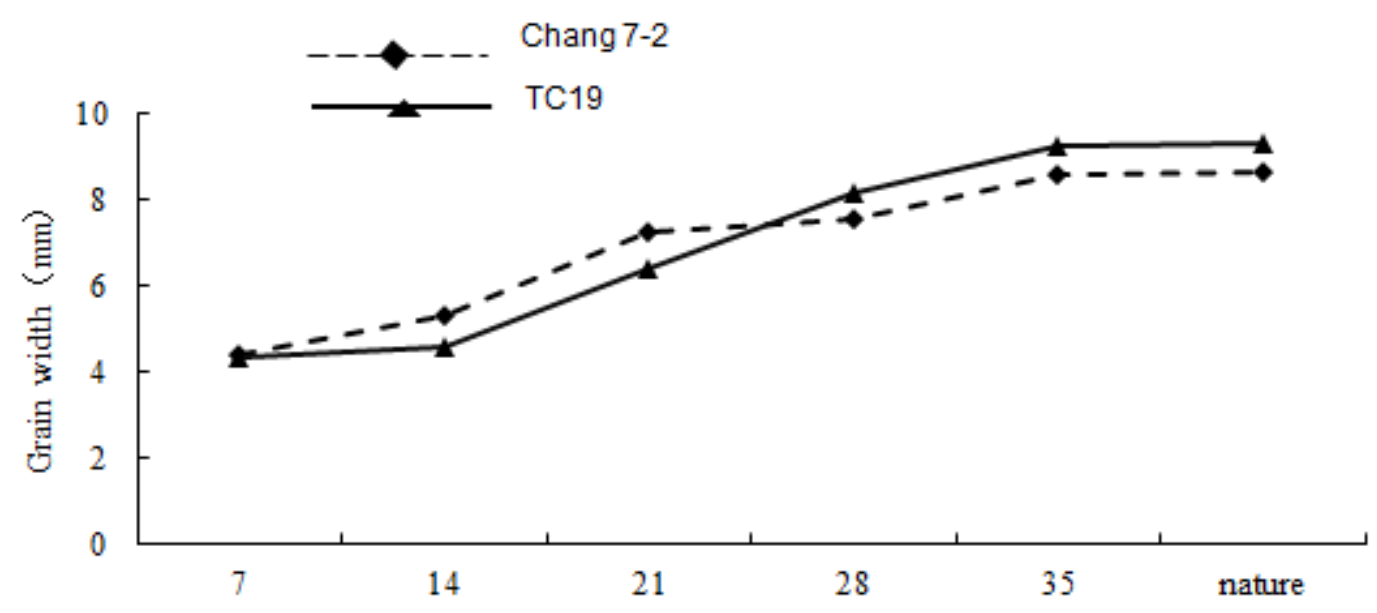

Figure 2

Grain size development of Chang 7-2 and TC19 at different days after pollination (DAP). A. Photograph showing the difference between Chang 7-2 and TC19 at different DAP. B. Statistics for grain width between Chang 7-2 and TC19 at different DAP. 

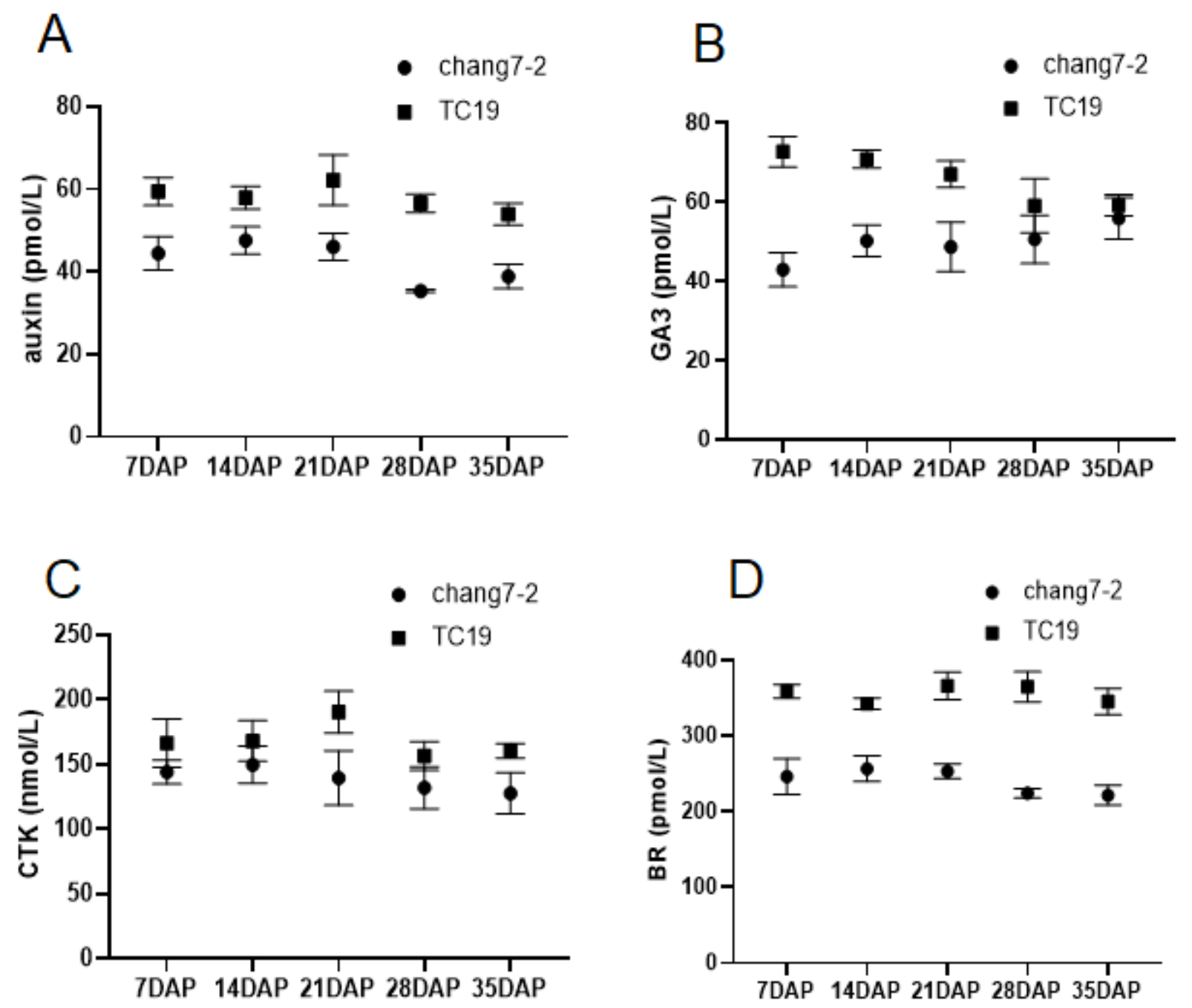

Figure 3

The concentration of hormones in different DAPs. A. the concentration of auxin at different DAP. B. The concentration of GA3 at different DAP. C. The concentration of CTK at different DAP. D. The concentration of $B R$ at different DAP. 
A

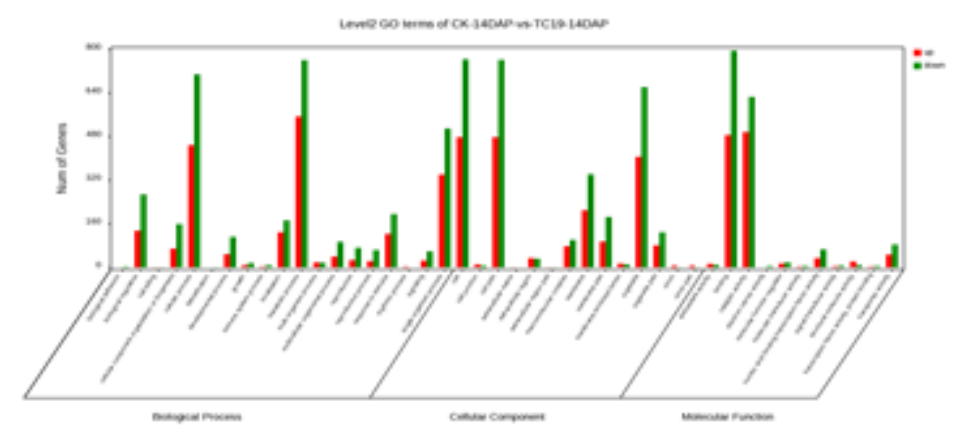

B

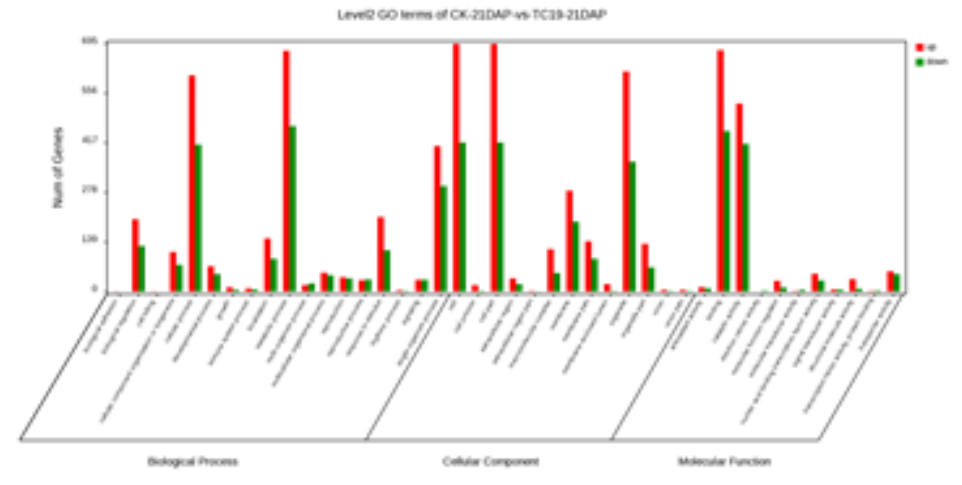

C

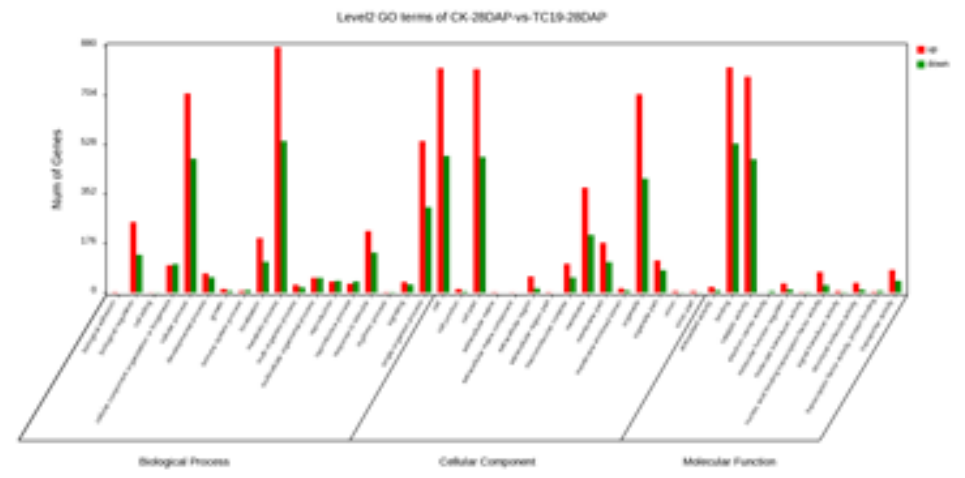

Figure 4

Analysis of KEGG enrichment at different DAP: A. 14 DAP, B. 21 DAP, and C. 28 DAP. 


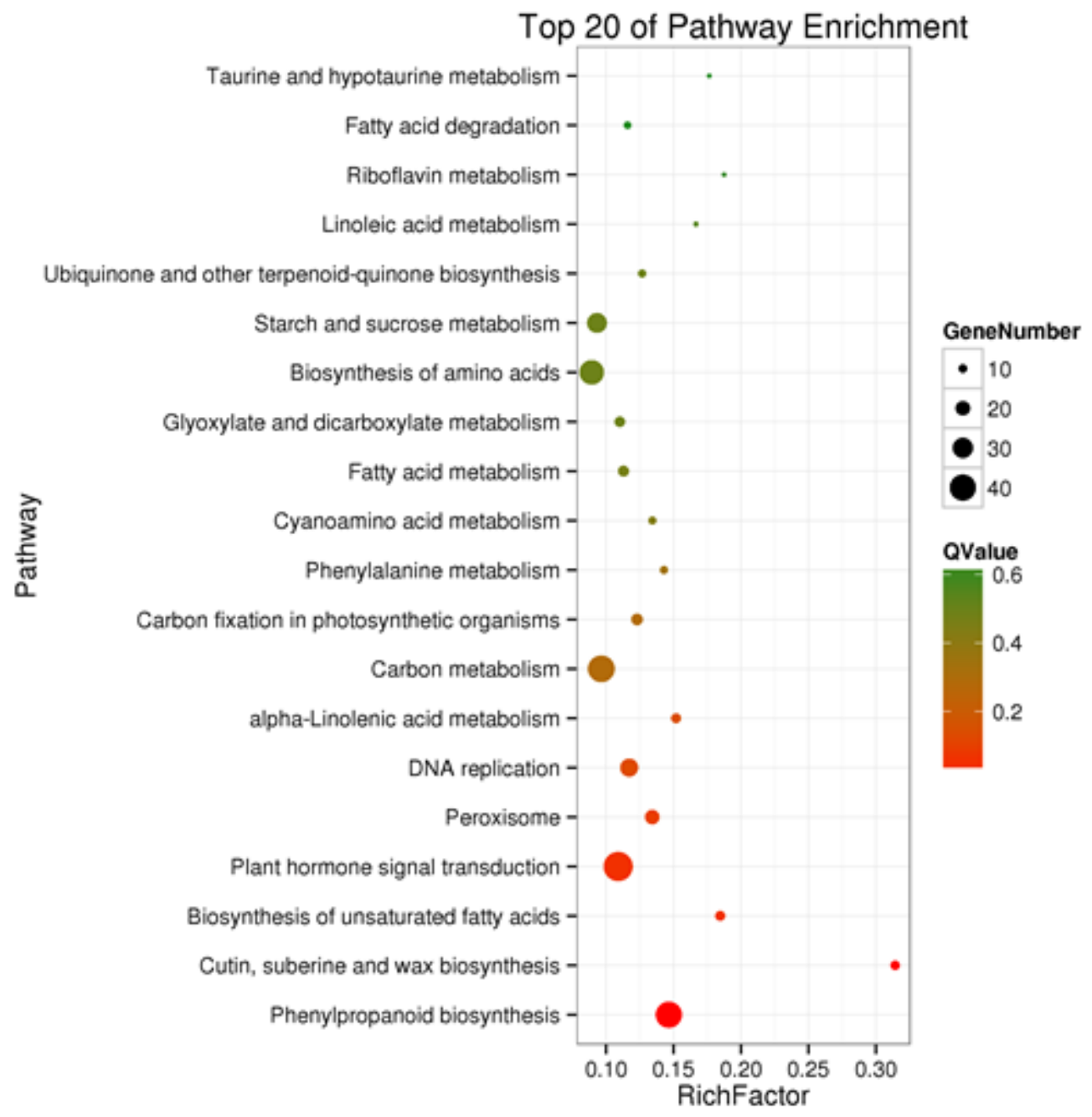

Figure 5

Analysis of pathway enrichment at 14 DAP. 


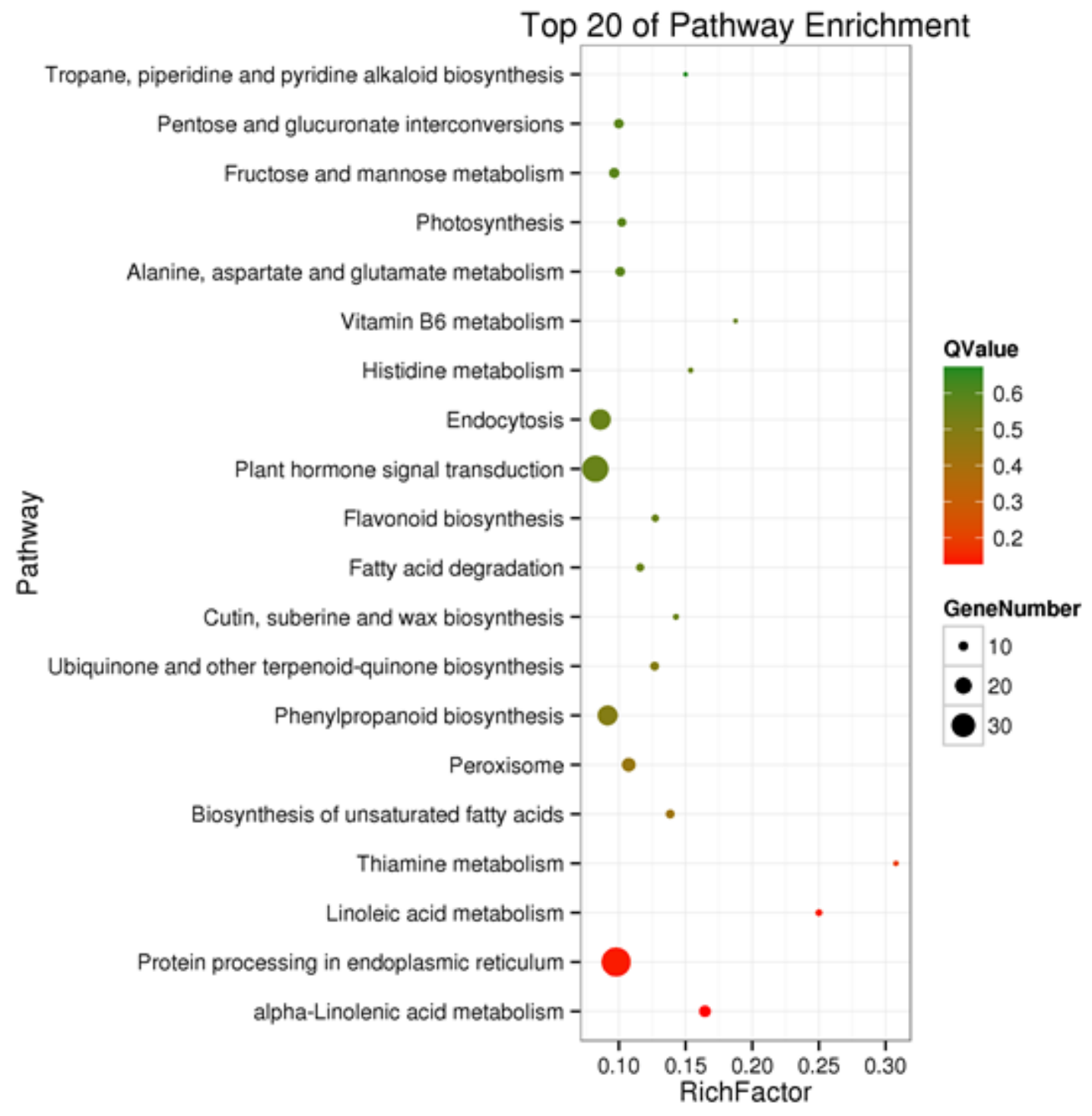

Figure 6

Analysis of pathway enrichment at 21 DAP. 


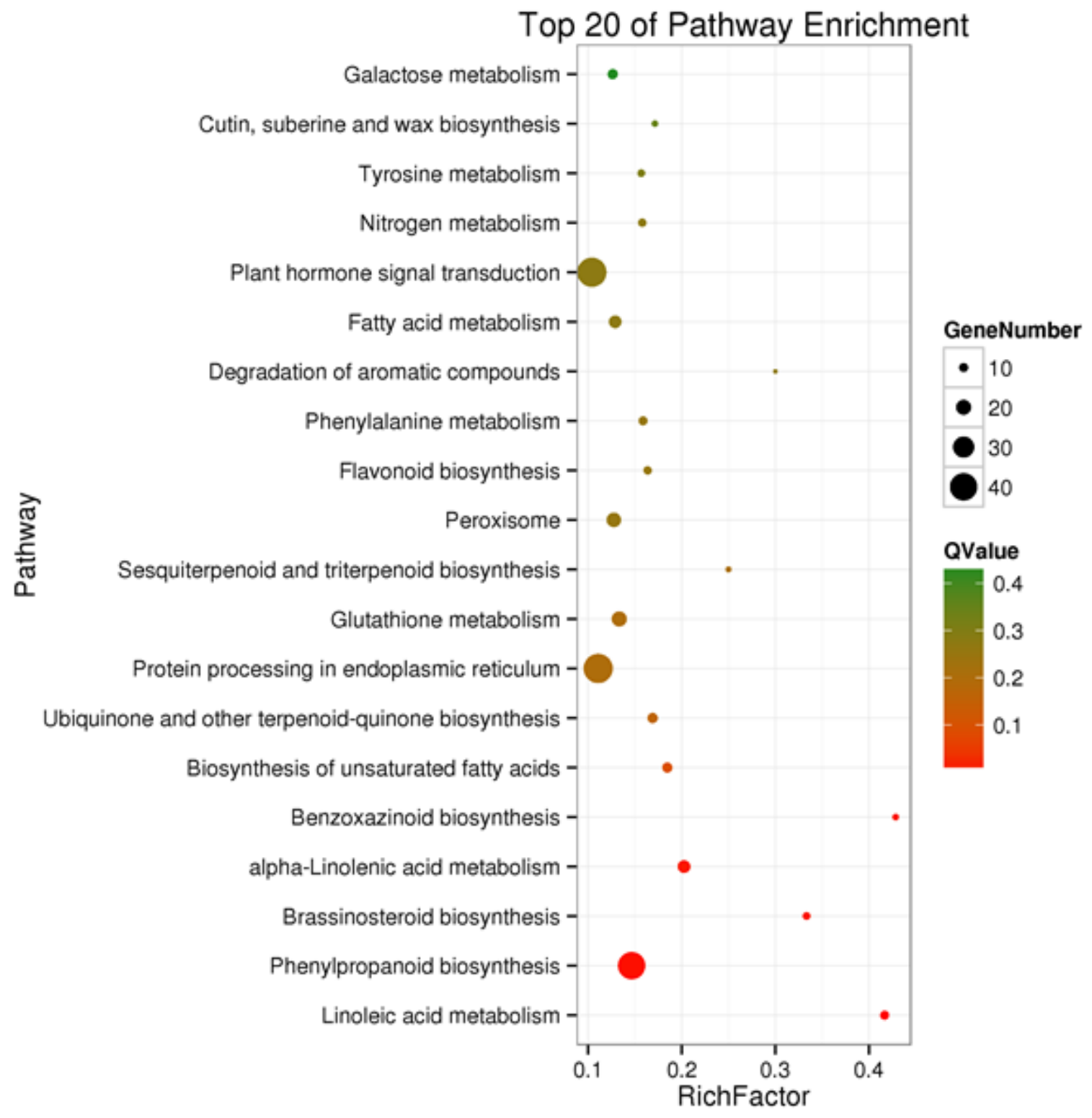

\section{Figure 7}

Analysis of pathway enrichment at 28 DAP. 
A

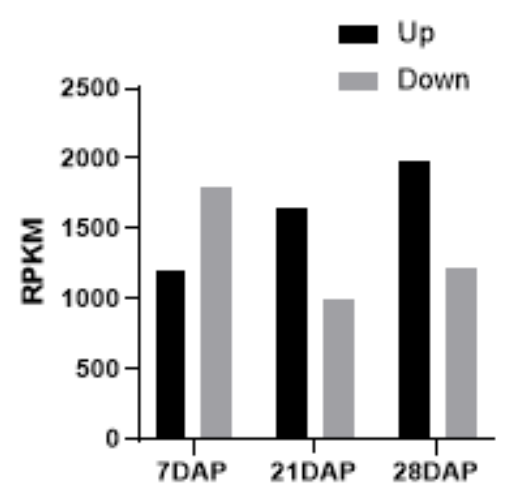

B

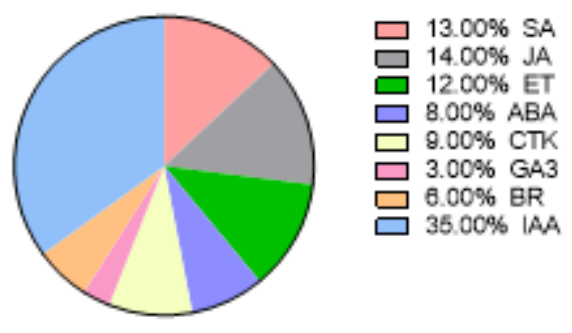

C

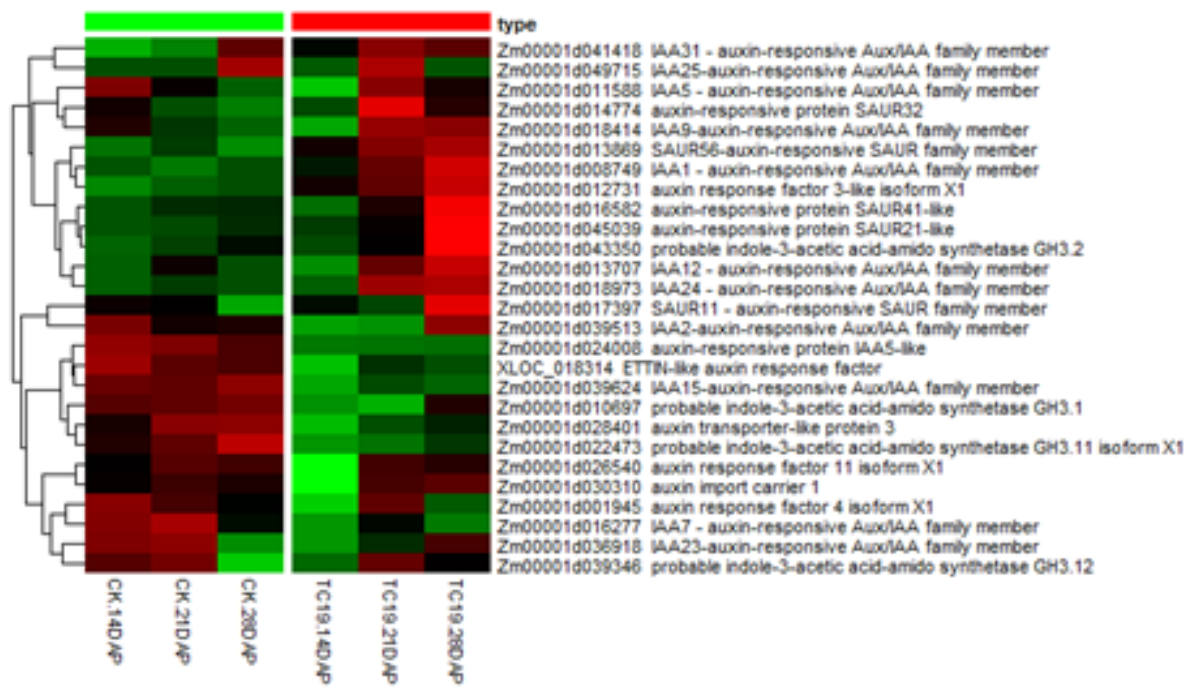

Figure 8

Statistics of the DEGs. A. DEG statistics. B. The distribution of hormone signaling pathway-related genes. C. The gene expression related to the IAA hormone signal transduction pathway. 

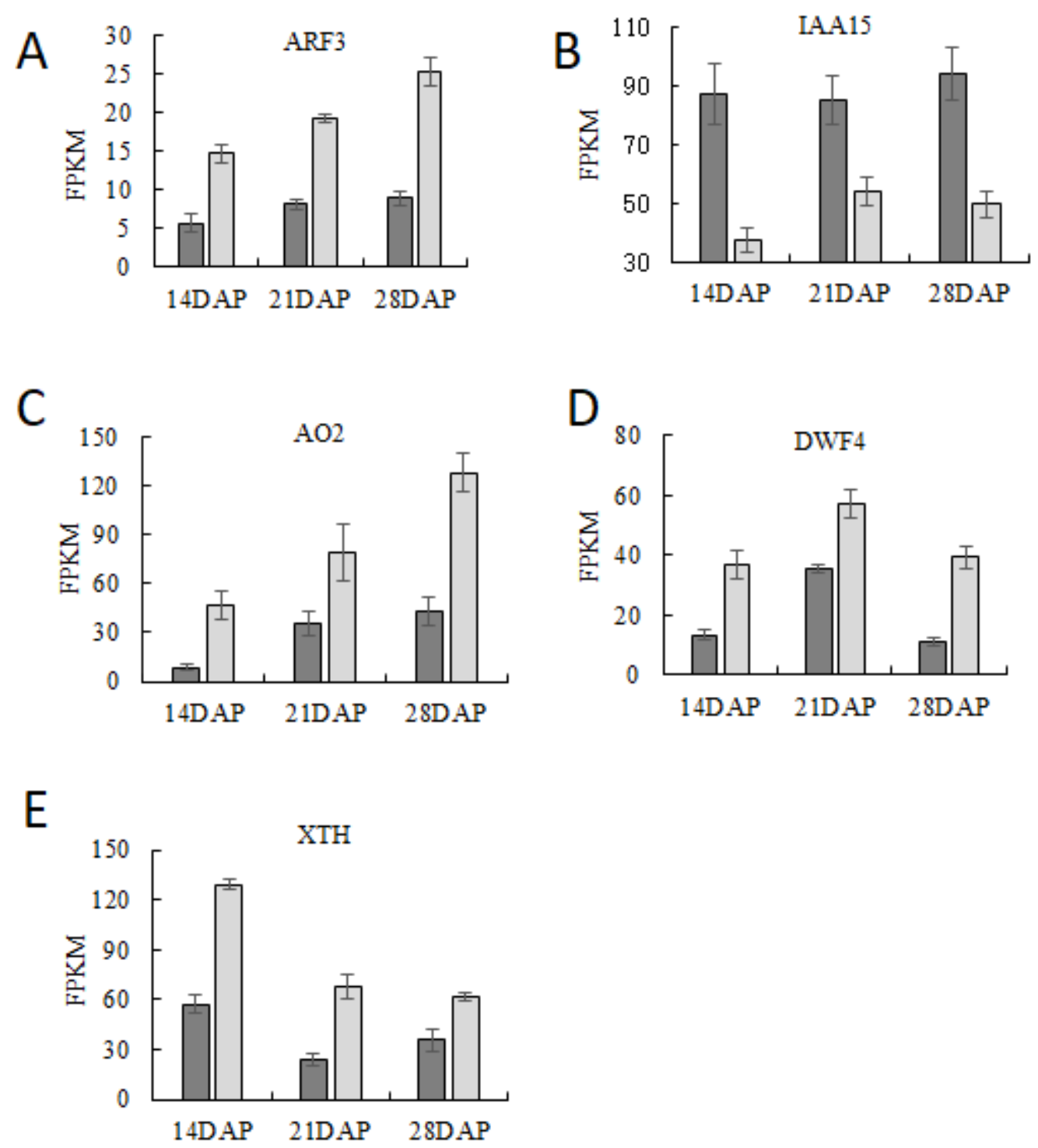

\section{Figure 9}

The gene expression of hormone-related genes. A. The expression of ARF3, B. The expression of IAA5, C. The expression of AO2, D. The expression of DWF4, and E. The expression of XTH. 


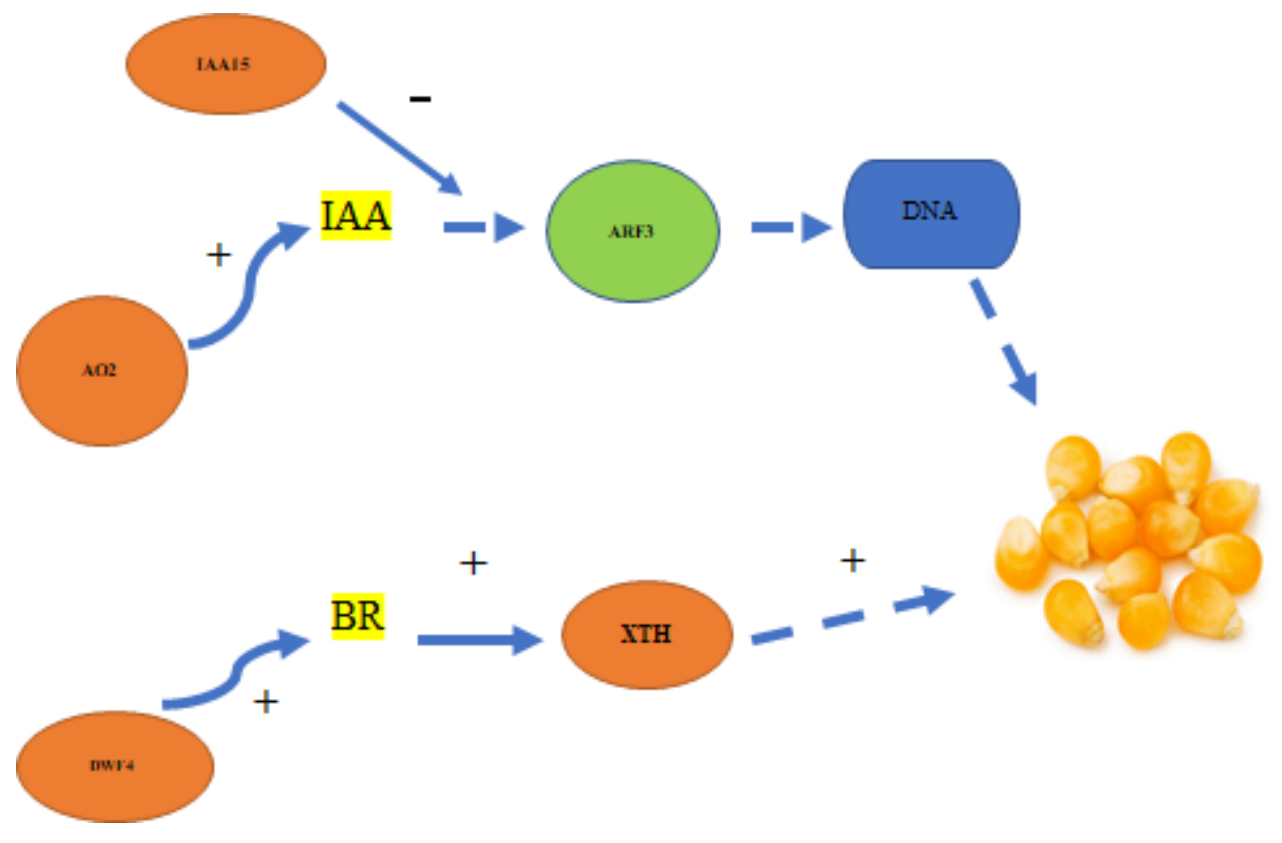

Figure 10

Proposed model for the grain size development mechanism.

\section{Supplementary Files}

This is a list of supplementary files associated with this preprint. Click to download.

- Additionalfile1tables1.docx

- additionalfile2tableS2.docx 